\title{
Preimplantation genetic testing for BRCA gene mutation carriers: a cost effectiveness analysis
}

Nadav Michaan ${ }^{1 *}$ (D) Moshe Leshno ${ }^{2}$, Yoni Cohen ${ }^{3}$, Tamar Safra ${ }^{4}$, Shira Peleg-Hasson ${ }^{4}$, Ido Laskov ${ }^{1 \dagger}$ and

Dan Grisaru ${ }^{1+}$

\begin{abstract}
Background: Gynecologic oncologists should be aware of the option of conception through IVF/PGT-M for families with high BRCA related morbidity or mortality. Our objective was to investigate the cost-effectiveness of preimplantation genetic testing for selection and transfer of BRCA negative embryo in BRCA mutation carriers compared to natural conception.

Methods: Cost-effectiveness of two strategies, conception through IVF/PGT-M and BRCA negative embryo transfer versus natural conception with a 50\% chance of BRCA positive newborn for BRCA mutation carriers was compared using a Markovian process decision analysis model. Costs of the two strategies were compared using quality adjusted life years (QALYs'). All costs were discounted at 3\%. Incremental cost effectiveness ratio (ICER) compared to willingness to pay threshold was used for cost-effectiveness analysis.

Results: IVF/ PGT-M is cost-effective with an ICER of 150,219 new Israeli Shekels, per QALY gained (equivalent to 44,480 USD), at a $3 \%$ discount rate.

Conclusions: IVF/ PGT-M and BRCA negative embryo transfer compared to natural conception among BRCA positive parents is cost effective and may be offered for selected couples with high BRCA mutation related morbidity or mortality. Our results could impact decisions regarding conception among BRCA positive couples and health care providers.
\end{abstract}

Keywords: IFV/PGT-M, BRCA, Cost-effectiveness, Ovarian cancer, Breast cancer

\section{Background}

BRCA mutation carriers have an estimated $80 \%$ life time risk of breast cancer, up to $40 \%$ life time risk of ovarian cancer, as well as increased risk of other malignancies including gastro-intestinal, pancreatic and prostate cancer [1]. In some family clusters, high

\footnotetext{
*Correspondence: nadavmi@gmail.com

${ }^{\dagger}$ Ido Laskov and Dan Grisaru contributed equally to this work.

${ }^{1}$ Gynecologic Oncology Department, Lis Maternity Hospital, Tel Aviv

Sourasky Medical Center, Sackler School of Medicine, Tel Aviv University, 6 Weismann st., 6296317 Tel Aviv, Israel
}

Full list of author information is available at the end of the article disease burden is noticed with several generations effected at young ages, causing significant physical and psychological morbidity $[2,3]$. Previous studies have shown that population based BRCA screening is cost effective and can be used as a screening tool that allows very effective risk reduction strategies for BRCA carriers [4-6]. Another possible strategy that may prevent passing on the BRCA gene to next generations is selection of BRCA negative embryos using in-vitro fertilization (IVF) and preimplantation genetic testing for monogenic/single gene disorders (PGT-M). PGT-M enables selection of unaffected embryos for embryo original author(s) and the source, provide a link to the Creative Commons licence, and indicate if changes were made. The images or other third party material in this article are included in the article's Creative Commons licence, unless indicated otherwise in a credit line to the material. If material is not included in the article's Creative Commons licence and your intended use is not permitted by statutory regulation or exceeds the permitted use, you will need to obtain permission directly from the copyright holder. To view a copy of this licence, visit http://creativecommons.org/licenses/by/4.0/. The Creative Commons Public Domain Dedication waiver (http://creativeco mmons.org/publicdomain/zero/1.0/) applies to the data made available in this article, unless otherwise stated in a credit line to the data. 
transfer and may be used for prevention of single gene disorders, such as BRCA gene mutations, in offspring $[7,8]$. The Ethics committee opinion of the European Society of Human Reproduction and Embryology stated that PGT-M for adult-onset conditions is ethically justifiable when the conditions are serious and when there are no known interventions for the conditions, or the available interventions are either inadequately effective or are perceived to be significantly burdensome [9]. Indeed, delivering a BRCA negative newborn would prevent the need for life long cancer surveillance for BRCA positive patients along with the medical, psychological and financial burden associated, and may be a suitable solution for some BRCA positive families.

The aim of our study was to investigate whether IVF/ PGT-M for BRCA negative embryo selection among BRCA positive parents as opposed to natural conception with a $50 \%$ chance of a BRCA positive fetus, due to dominant gene inheritance, would be a cost effective strategy.

\section{Methods}

The target population for our research are potential BRCA positive parents (mother or father). Costs of IVF/ PGT-M, with BRCA negative embryo selection and transfer versus natural conception with a $50 \%$ chance of BRCA positive newborn, were compared using a Markovian process decision analysis model (Fig. 1). The model assumed that all women in the IVF/ PGT-M arm would undergo ovarian stimulation and ovum pick. After intracytoplasmic sperm injection (ICSI), fertilization and embryo biopsy, a BRCA negative embryo would be selected for embryo transfer. In the natural conception arm, women would conceive naturally, without any manipulation, and assume a $50 \%$ chance of bearing a BRCA positive newborn, as BRCA is a dominant gene. BRCA negative newborns would assume to have the general populations' life-time-risk of breast and ovarian cancer. At age 40, BRCA positive females would be offered risk reduction salpingo-oophorectomy (RRSO) for ovarian cancer prevention [10]. Management of breast cancer

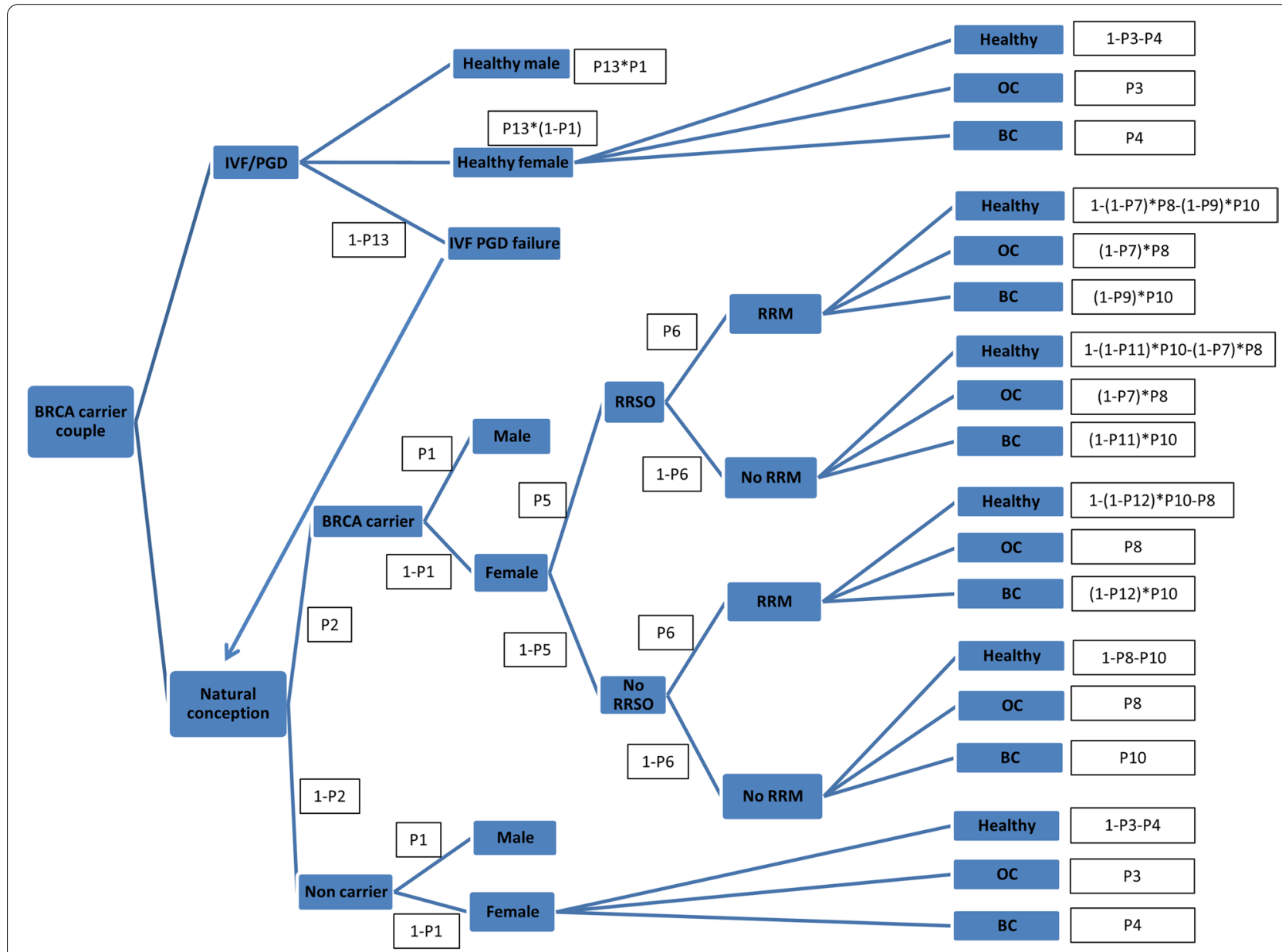

Fig. 1 Markovian decision analysis model, IFV/PGD for BRCA mutation carriers versus natural conception 
risk for BRCA positive females would include screening with yearly breast MRI/ultrasound or risk reduction mastectomy (RRM) for breast cancer prevention [10]. Costs of these two strategies were compared using qualityadjusted life years (QALYs), which reflect both quality and quantity of life lived. Incremental cost effectiveness ratio (ICER) was used for cost-effectiveness analysis, compared to a willingness to pay threshold.

\section{IVF/PGT-M arm}

In this arm, all couples with either one BRCA positive parent were assumed to undergo ovarian stimulation, ovum pick-up and ICSI. After fertilization, embryos would be biopsied and only BRCA negative embryos would be transferred. All women were assumed to have IVF before age 35, where IVF success rates are optimal [11], as the knowledge of BRCA carrier state among afflicted families would to be known at an early age. The following steps were assumed in the IVF/ PGT-M arm, probabilities were taken from the ESHRE PGD consortium data collection regarding success of IVF/ PGT-M cycles preformed for single, autosomal dominant gene disorders [8]: a: ovarian stimulation and ovum pickup, on average, 13 oocytes are retrieved per IVF cycle among patients $<35$ years, undergoing IVF/PGD for dominant, single gene disorders, b: insemination with ICSI, $82 \%$ of oocytes are successfully inseminated, c: fertilization, $76 \%$ of inseminated oocytes are fertilized, d: embryo biopsy, $79 \%$ of embryos are successfully biopsied.

Accordingly, per cycle start, out of 13 oocytes retrieved, 6.4 embryos will be available for biopsy, half of which, 3.20 embryos, will be BRCA negative embryos, available for transfer. The first embryo will be used for fresh embryo transfer, the remaining embryos will be frozen for future frozen thawed embryo transfer cycles.

The overall live birth rate per embryo transfer for couples undergoing IVF/ PGT-M for genetic disorders reaches $45.8 \%$ [12]. The model assumed that after the first fresh embryo transfer, $54.2 \%$ of couples who fail would have a second thawed embryo transfer, while $29 \%$ of couples who will fail the second transfer, will have a third, thawed embryo transfer. Therefore, 3 available healthy, non BRCA mutated embryos, with a $45.8 \%$ live birth rate per embryo transfer would result in an overall $84 \%$ chance of a live, BRCA negative baby, from one cycle of fresh embryo transfer and two more cycles of thawed embryo transfers. The remaining $16 \%$ of couples who failed the IVF/ PGT-M path would go back to the natural conception arm.

\section{Natural conception arm}

In this arm, couples are assumed to conceive naturally. As BRCA is a dominant gene, these couples would have a $50 \%$ chance of bearing a BRCA positive fetus, of those, $50 \%$ would be females, positive for BRCA gene mutations with respective increased risk of breast and ovarian cancer. As the chances of other BRCA related malignancies among male mutation carriers are much lower than among female mutation carriers these were not included in our model. BRCA negative newborns were assumed to have the natural populations' breast and ovarian cancer risk.

\section{Model costs}

In vitro fertilization, PGT-M and embryo transfer, as well as BRCA screening and other treatment related costs including, RRSO, RRM and breast and ovarian cancer treatment costs were received from the Israeli ministry of health 2020 pricing list according to specified codes with conservative assumptions of health resources utilities. Elaborate costs used in the model, from payer perspective, are shown in supplementary Table 1, 2, 3, 4 and 5 for, ovarian cancer treatment, breast cancer treatment, IVF/PGT-M, BRCA positive patients follow-up and total costs, respectively. All costs were discounted at $3 \%$.

\section{Probabilities}

Model probabilities are presented in Table 1. The probability of being at the end of each arm of the Markovian model was calculated by multiplying the probabilities of events along the arms' path. Stage distribution and Kaplan Meir survival curves for each stage were used to calculate mortality rates of subjects who developed breast cancer or ovarian cancer, based on the Surveillance, Epidemiology and End Results program data base (SEER) $[13,14]$. Survival curves were extrapolated by fitting Weibull distribution using the Nelder-Mead Algorithm.

\section{Quality adjusted life years and incremental cost effectiveness ratio}

Value of health benefits for each strategy (IVF/ PGT-M versus natural conception) were calculated using quality adjusted life years (QALY's). QALY's are calculated by multiplying the utility value associated with a given state of health by the number of years lived in that state, where QALY of one reflects 1 year lived in perfect health and QALY of zero represents death state. The incremental cost effectiveness ratio was then calculated by using the formula: (average cost IVF/ PGT-M - average cost natural conception) / (average QALY IVF/ PGT-M - average QALY natural conception). The incremental cost effectiveness ration (ICER) calculated, enabled to determine whether offering IVF/ PGT-M to BRCA positive families is cost effective or not, compared to willingness to pay threshold. An intervention was defined as cost effective if the ICER 
Table 1 Probabilities used in model

\begin{tabular}{|c|c|c|c|}
\hline Probability & Description & Probability & Range assumed \\
\hline P1 & Probability of male newborn & 0.5 & \\
\hline P2 & Probability newborn is BRCA carrier & 0.5 & \\
\hline P3 & Probability that a non-carrier will experience ovarian cancer ${ }^{13}$ & 0.0128 & $0.0005-0.0989$ \\
\hline P4 & Probability that a non- carrier will experience breast cancer ${ }^{14}$ & 0.13 & $0.11-0.14$ \\
\hline P5 & Probability that carrier will undergo RRSO 20,21 & 0.65 & $0.3-0.75$ \\
\hline P6 & Probability that BRCA carrier will undergo RRM ${ }^{25}$ & 0.16 & $0.13-0.3$ \\
\hline P7 & Reduction in risk of ovarian cancer from RRSO 26,27 & 0.8 & $0.8-0.96$ \\
\hline P8 & Probability that BRCA carrier without RRSO will get OC ${ }^{28}$ & 0.2987 & $0.24-0.35$ \\
\hline P9 & Reduction in risk of breast cancer from RRM and $\mathrm{RRSO}^{27}$ & 0.91 & $0.78-0.99$ \\
\hline P10 & Probability that a BRCA carrier without RRM will experience breast cancer ${ }^{28}$ & 0.53 & $0.44-0.62$ \\
\hline P11 & Reduction in risk of breast cancer from RRSO 22,24,29 & 0.0 & $0.37-0.65$ \\
\hline P12 & Reduction in breast cancer risk from RRM without RRSO ${ }^{30}$ & 0.91 & $0.62-0.98$ \\
\hline P13 & $\begin{array}{l}\text { Probability of live newborn with IVF/PGD ( } 1 \text { cycles of fresh ET and } 2 \text { cycles } \\
\text { of thawed ET) }\end{array}$ & 0.84 & $0.7-0.9$ \\
\hline
\end{tabular}

RRSO Risk reduction salpingo-oophorectomy, RRM Risk reduction mastectomy, IVF In-vitro fertilization, PGD Pre-gestational diagnosis, ET Eembryo transfer

per QALY is between 1 and 3 times per capita gross national product (GNP). GNP in Israel is estimated at 42,160 USD, equivalent to 142,500 new Israeli Shekels (NIS) [15]. Interventions below 1 GNP per capita are considered very cost effective [16].

\section{Sensitivity analysis}

One-way sensitivity analysis was conducted with all variables to evaluate model uncertainties. In addition, a probabilistic sensitivity analysis (Monte Carlo simulation) was conducted with all variables, using 100 trials, each included 10,000 couples.

\section{Results}

Delivering a BRCA negative newborn after IVF/ PGT$\mathrm{M}$, compared to natural conception, is cost effective according to our model, with an ICER of 150,219 NIS per QALY gained, at a 3\% discount rate compared to a willingness to pay threshold of 1-3 times Israeli GNP per capita, equivalent to 44,480 USD (Table 2).

\section{Sensitivity analysis}

In order to assess the effect of each parameter on the ICERS, a sensitivity analysis was conducted. Results are shown on a tornado diagram (Fig. 2). The most influential parameter that effects the ICER was found to be the discount rate, set at 3\% in our model. As the money spent on IFV/ PGT-M is used at present, while screening strategies for BRCA carriers, risk reduction surgeries as well as breast and ovarian cancer treatments start many years later (beginning at age 30 years), discounting makes costs of future spending much lower than current values. Reducing the discount rate to zero resulted in a negative ICER of - 19,658 NIS, meaning that IVF/ PGT-M would be cost saving and not just cost effective.

Probabilistic sensitivity analysis (Monte Carlo simulation) was conducted with all variables, using 100 trials, each included 10,000 couples. At a willingness to pay threshold of 340,000 NIS (which are equivalent to 100,000 USD), in over $85 \%$ of simulations iterations IVF/ PGT-M compared to natural conception is cost effective (Acceptability curve, which shows the results of probabilistic sensitivity analysis and presents the relative

Table 2 Cost effectiveness analysis of IVF/PGD versus natural conception for BRCA negative embryo selection

\begin{tabular}{lcccc}
\hline Strategy & Cost (NIS) & Incremental cost & QALY & Incremental QALY \\
\hline 3\% discount rate & & & 30.1359 \\
IVF/PGD & 12,057 & 18,811 & 30.2611 & 0.1252 \\
Natural conception & 30,868 & & & 77.7 \\
Not discounted (0\% discount rate) & 49,767 & 0 & 76.8 & 0 \\
IVF/PGD & 66,809 & 17,041 & -0.866 \\
Natural selection & & & 150,219 \\
\hline
\end{tabular}

IVF In vitro-fertilization, PGD Preimplantation genetic diagnosis, QALY Quality adjusted life years, ICER Incremental cost effectiveness ration, NIS New Israeli Shekels 


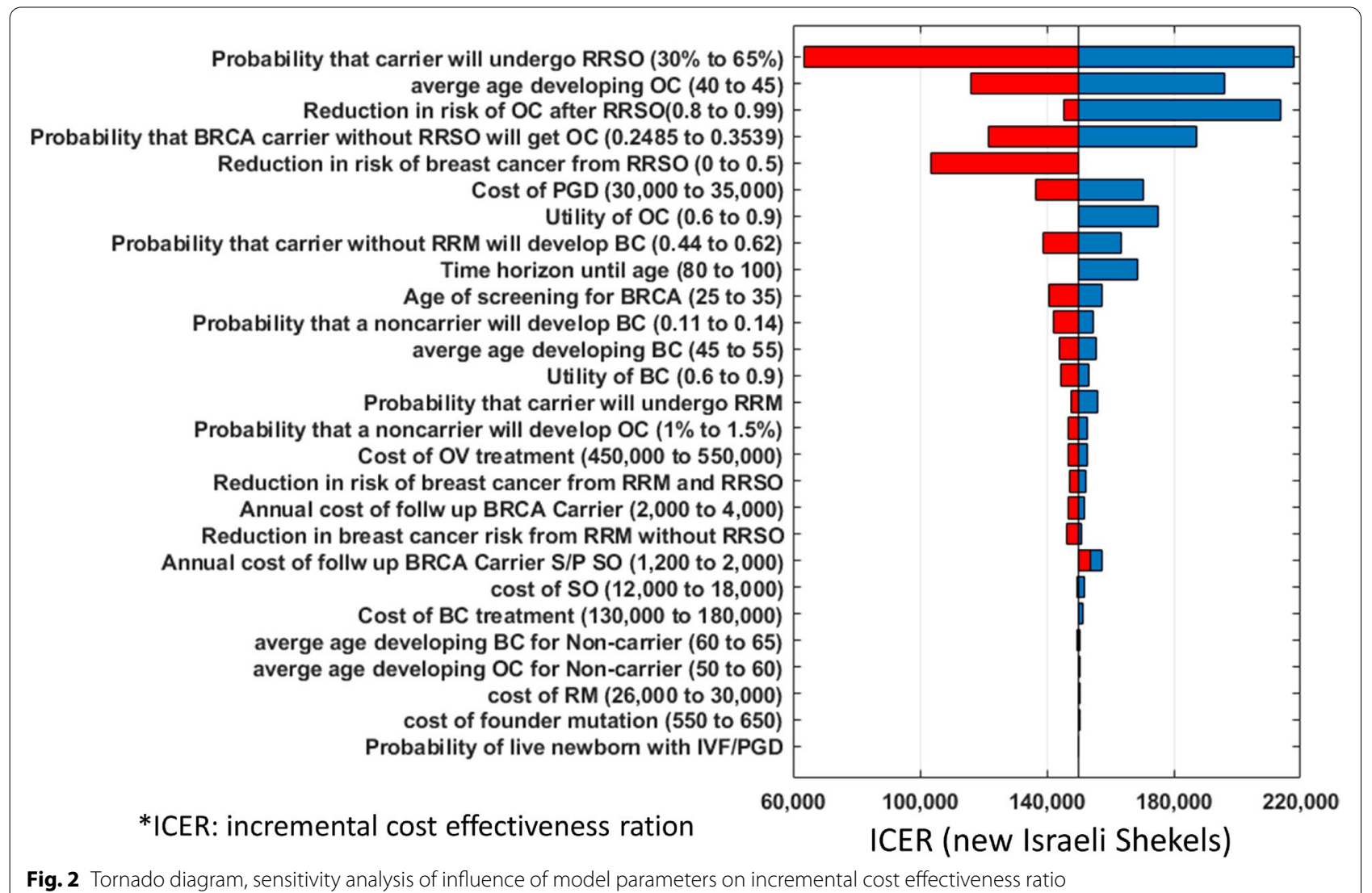

Fig. 2 Tornado diagram, sensitivity analysis of influence of model parameters on incremental cost effectiveness ratio

cost-effectiveness as a function of the ICER, willingness to pay threshold, is shown in Supplementary Fig. 1).

\section{Discussion}

In this cost effectiveness analysis, we found that at current pricing, with a $3 \%$ discount rate, IVF/ PGT-M for BRCA negative embryo selection is cost effective compared to natural conception, with an ICER of 150,219 NIS per QALY gained. This ICER is just short of being very cost effective, compared to a WTP threshold of one GNP per capita in Israel.

Advances in PGT-M can prevent passing of single gene disorders such as BRCA mutations to future generations by selecting a BRCA negative embryo. The prevalence of BRCA gene mutations reaches as high as 2.5\% among Jews of Ashkenazi ancestry [17] and although offering IVF/ PGT-M on a large population scale may not be feasible, this technology can be an extremely useful solution for families with very high BRCA related morbidity or mortality. These families carry a heavy medical as well as psychological burden with a life-long fear of cancer as well as fear of passing this cancer potential to their descendants. Our model shows that IVF/PGT-M is a cost effective option that may be offered to selected patients. This option should be kept in mind of gynecologists, gynecologic oncologists, oncologist and any other physician involved with medical care of BRCA positive patients.

The sensitivity analysis showed that the most important factor impacting the ICER in our model is the discount rate. This is caused because the costs involved in IVF/ PGT-M are assumed to be spent at present while the money saved by preventing the birth of a BRCA positive offspring will only be seen within 30-40years when screening and risk reduction surgeries will be implemented. Thus, the long projection period makes the yearly discount a very influential factor in the model. Reducing the discount rate to zero resulted in a negative ICER (-19,658 NIS), making IVF/PGT-M cost saving. In a similar work, only recently published by Lipton et-al, a more conservative discount rate of $1.5 \%$ was assumed [18]. In this work, that was done on Canadian data, IVF/ PGT-M was found to be cost effective for both BRCA1 and BRCA2 mutation carriers with an ICER of 14,242 USD and 12,893 USD, respectively. In our model the base line discount rate assumed was $3 \%$, one that is used more frequently in cost effectiveness analysis in the United States and in most European countries [19]. 
The probability that a BRCA carrier would undergo RRSO also had a significant influence on the ICER calculated (tornado diagram, Fig. 2). The probability estimated in our model was $65 \%$, based on previous data $[20,21]$, raising this probability would make the ICER significantly more cost effective. Presuming that IFV/PGT-M would be offered to families with high disease burden, we believe that the uptake of both RRSO and RM would be much higher among these families, making IFV/PGT-M even more cost effective. The least influential factor that effects the ICER was found to be the probability of a live, BRCA negative baby after IVF/PGT-m (tornado diagram, Fig. 2). In our model this was calculated to be $84 \%$ after one round of fresh embryo transfer and two more rounds of thawed embryo transfer for women under age 35 . IVF/PGT-m success rates may undoubtedly differ between clinics, techniques, protocols, experience and expertise and these differences are reflected in different reports regarding IVF success rates. Yet our model shows that even a large overestimation or underestimation of IVF/PGT-m success rates has little effect on the ICER. Even doubling the percent of couples that would fail to deliver a BRCA negative baby through IVF/PGT-m and that would need to go back and conceive naturally after unsuccessful IVF/PGT-m from 16 to $30 \%$ was found to hardly effect the ICER calculated (Fig. 2).

Our work has several limitations. As corralation between BRCA gene mutation and other malignancies, such as prostate, gastrointestinal and pancreatic cancer is much weaker we decided not to include these in our model. The number of uncertanties that would arise in a model that would include many more malignancies would be, in our opinion, too large, making our theoretical model much weaker. Another major limitation that would need to be addressed as IVF and PGT-M technologies improve, making it more readily available for widespread use is the ethical justification of putting couples through a potentially dangerous medical procedure in order to avoid potential disease that would develop many years later such as ovarian cancer that is highly preventable with RRSO and breast cancer that is both preventable and highly curable for patients under tight follow-up. From a practical standpoint, the prevalence of BRCA in Israel, particularly among Jews of Askenazi origin, is as high as $2.5 \%$, and offering IVF/ PGT-M on a large population scale might not be a feasible option. Saving this procedure to selected families with high disease burden or extreme cancer anxiety would be a more practical approach.

Our work has several advantages. In our model, IVF/ PGT-M strategy was found to be cost effective compared to natural conception. When calculating the costs of breast and ovarian cancer treatment, we assumed a very conservative use of health resource utilities. In addition, costs involved with male BRCA related malignancies were not included in our calculations, (mainly because the probabilities of these malignancies are much smaller). Had we taken these risks into consideration in our model, cost-effectiveness would likely to increase considerably. Another important point that underestimates the advantage of having IVF/ PGT-M, and would make this strategy more cost effective, is the elimination of the $50 \%$ chance of passing on the BRCA gene to second generations in the IVF/ PGT-M arm. In the natural conception arm, BRCA positive newborns would themselves have a $50 \%$ chance of passing the BRCA mutated gene to their offspring with the costs involved. Our model included the most up-to-date recommendations for breast and ovarian cancer treatments including the use of poly ADP ribose polymerase (PARP) inhibitors for the first line treatment of ovarian cancer, that was only recently approved for use in Israel, in BRCA positive patients. These are highly costly drugs that add considerably to cancer treatment costs. Most up-to-date data regarding the effect of RRSO on breast cancer risk was also included in our model. Previous data estimated a 50\% reduction in risk of breast cancer as a result of RRSO. Newer data that used RRSO as a time dependent covariate did not find such an effect and in fact found no influence of RRSO on breast cancer risk [22-24]. A possible strategy that would further increase success rates of IVF/PGT-M, for couples who failed to produce a BRCA negative embryo would be to transfer a male embryo, regardless of its' BRCA status. Using a male BRCA positive embryo for transfer would not prevent passing on the BRCA gene to the next generation, but would eliminate the risk of ovarian cancer, keep the chance of breast cancer (male breast cancer) very low while increasing the number of embryos available for transfer.

\section{Conclusion}

IVF/PGT-M for BRCA positive parents versus natural conception at current pricing, with a $3 \%$ discount rate, is cost effective and should be offered to selected couples. Discount rate is the most influential parameter that effects the ICER.

\section{Abbreviations}

IVF: In-vitro fertilization; PGT-M: Preimplantation genetic testing; ICSI: Intracytoplasmic sperm injection; RRSO: Risk reduction salpingo-oophorectomy; RRM: Risk reduction mastectomy; QALYs: Quality-adjusted life years; ICER: Incremental cost effectiveness ratio; GNP: Gross national product; NIS: New Israeli Shekels. 


\section{Supplementary Information}

The online version contains supplementary material available at https://doi. org/10.1186/s12958-021-00827-9.

Additional file 1: Supplementary Table 1: Ovarian cancer diagnosis and treatment costs.

Additional file 2: Supplementary Table 2: Breast cancer diagnosis and treatment costs (All costs received from $\mathrm{IMH}^{*}$ pricing list (1).

Additional file 3: Supplementary Table 3: Yearly BRCA carrier follow up costs (All costs received from IMH* pricing list [1] according to NCCN surveillance guidelines (2).

Additional file 4: Supplementary Table 4: IVF PGD costs used in model, according to Israeli ministry of health (IMH) pricing list ${ }^{1}$ (1 fresh round +2 thawed rounds)*.

Additional file 5: Supplementary Table 5: Total costs used in model.

Additional file 6. Acceptability curve.

\section{Acknowledgements}

Not applicable.

\section{Authors' contributions}

All authors contributed substantially to planning and caring out this work. NM: main author, involved in conceptualization, methodology, project administration, investigation, data analysis, supervision, writing - original draft, and writing - review and editing. ML: involved in conceptualization, methodology, project administration, investigation, data analysis, supervision, writing - original draft, and writing - review and editing. TS: Involved in methodology, project administration, data curation and analysis, supervision, reviewing original draft. YC: Involved in conceptualization, data curation, investigation, supervision, and writing of original draft. SPH involved in conceptualization, data curation, investigation, supervision, and writing of original draft. IL: Involved in conceptualization, methodology, project administration, data curation, supervision, writing - original draft and reviewing. GD: Involved in Conceptualization, methodology, project administration, investigation, data curation and analysis, supervision, writing - original draft and reviewing. The authors read and approved the final manuscript.

\section{Funding}

Not applicable, this work received no funding.

\section{Availability of data and materials}

Not applicable (theoretical mathematical model).

\section{Declarations}

\section{Ethics approval and consent to participate}

After consultation with our local institutional review board of the Tel-Aviv Sourasky medical Center, as this work is a theoretical, mathematical model, not involving any human or animal subjects in any form, it was exempt from the need of IRB approval.

\section{Consent for publication}

Not applicable.

\section{Competing interests}

The authors declare that they have no competing interests

\section{Author details}

${ }^{1}$ Gynecologic Oncology Department, Lis Maternity Hospital, Tel Aviv Sourasky Medical Center, Sackler School of Medicine, Tel Aviv University, 6 Weismann st., 6296317 Tel Aviv, Israel. ${ }^{2}$ Gastro-enterology, Tel Aviv Sourasky Medical Center, Coller School of Management and Sackler School of Medicine, Tel Aviv University, Tel Aviv, Israel. ${ }^{3}$ In-vitro Fertilization Unit, Lis Maternity Hospital, Tel Aviv Sourasky Medical Center, Sackler School of Medicine, Tel Aviv University, Tel Aviv, Israel. ${ }^{4}$ Oncology Department, Tel Aviv Sourasky Medical Center, Sackler School of Medicine, Tel Aviv University, Tel Aviv, Israel.
Received: 26 July 2021 Accepted: 6 September 2021

Published online: 08 October 2021

\section{References}

1. Wooster R, Weber BL. Breast and ovarian cancer. Guttmacher AE, Collins FS, editors. N Engl J Med. 2003;348:2339-47.

2. Shenfield F, Pennings G, Devroey P, Sureau C, Tarlatzis B, Cohen J, et al. Taskforce 5: Preimplantation genetic diagnosis. Hum Reprod. 2003;18:649-51.

3. Daum H, Peretz T, Laufer N. BRCA mutations and reproduction. Fertil Steril. 2018;109:33-8.

4. Manchanda R, Patel S, Antoniou AC, Levy-Lahad E, Turnbull C, Evans DG, et al. Cost-effectiveness of population based BRCA testing with varying Ashkenazi Jewish ancestry. Am J Obstet Gynecol. 2017;217:578. e1-578.e12.

5. Manchanda R, Loggenberg K, Sanderson S, Burnell M, Wardle J, Gessler S, et al. Population testing for cancer predisposing BRCA1/BRCA2 mutations in the ashkenazi-jewish community: a randomized controlled trial. J Natl Cancer Inst. 2015;107:1-11.

6. Michaan N, Leshno M, Safra T, Sonnenblick A, Laskov I, Grisaru D. Cost effectiveness of whole population BRCA genetic screening for cancer prevention in Israel. Cancer Prev Res (Phila). 2021:14(4):455-62.

7. Sharpe A, Avery P, Choudhary M. Reproductive outcome following pre-implantation genetic diagnosis (PGD) in the UK. Hum Fertil. 2018:21:120-7.

8. De Rycke M, Goossens V, Kokkali G, Meijer-Hoogeveen M, Coonen E, Moutou C. ESHRE PGD consortium data collection XIV-XV: cycles from January 2011 to December 2012 with pregnancy follow-up to October 2013. Hum Reprod. 2017:32:1974-94.

9. Daar J, Benward J, Collins L, Davis J, Davis O, Francis L, et al. Use of preimplantation genetic testing for monogenic defects (PGT-M) for adult-onset conditions: an ethics committee opinion. Fertil Steril. 2018:109:989-92.

10. NCCN clinical practice guidlines in oncology. Genetic/Familial High Risk Assessment: Breast, Ovarian and Pancreatic [Internet]. 2020 Version 2.2021 - Novemb. [cited 2020 Jul 4]. Available from: https://www.nccn. org/professionals/physician_gls/pdf/genetics_bop.pdf

11. Department of Health U, Services Centers for Disease Control H. 2016 Assisted Reproductive Technology National Summary Report [Internet] 2016. Available from: https://www.cdc.gov/art/reports/

12. Chang J, Boulet SL, Jeng G, Flowers L, Kissin DM. Outcomes of in vitro fertilization with preimplantation genetic diagnosis: an analysis of the United States assisted reproductive technology surveillance data, 2011-2012. Fertil Steril. 2016:105:394-400.

13. Ovarian Cancer - Cancer Stat Facts [Internet]. [cited 2019 Dec 14]. Available from: https://seer.cancer.gov/statfacts/html/ovary.html

14. Female Breast Cancer - Cancer Stat Facts [Internet]. [cited 2019 Dec 14]. Available from: https://seer.cancer.gov/statfacts/html/breast.html

15. Israel - OECD Data [Internet]. [cited 2020 Oct 30]. Available from: https:// data.oecd.org/israel.htm

16. Ginsberg GM, Fisher M, Ben-Shahar I, Bornstein J. Cost-utility analysis of vaccination against HPV in Israel. Vaccine. 2007;25:6677-91.

17. Levy-Lahad E, Catane R, Eisenberg S, Kaufman B, Hornreich G, Lishinsky E, et al. Founder BRCA1 and BRCA2 mutations in Ashkenazi Jews in Israel: frequency and differential penetrance in ovarian cancer and in breastovarian cancer families. Am J Hum Genet. 1997;60:1059-67 Available from: http://www.ncbi.nlm.nih.gov/pubmed/9150153\%0A http://www. pubmedcentral.nih.gov/articlerender.fcgi?artid=PMC1712434.

18. Lipton JH, Zargar M, Warner E, Greenblatt EE, Lee E, Chan KKW, et al. Cost effectiveness of in vitro fertilisation and preimplantation genetic testing to prevent transmission of BRCA1/2 mutations. Hum Reprod. 2020;35:434-45.

19. Attema AE, Brouwer WBF, Claxton K. Discounting in economic evaluations [internet]. Pharmacoeconomics. 2018:36(7):745-58.

20. Kram V, Peretz T, Sagi M. Acceptance of preventive surgeries by Israeli women who had undergone BRCA testing. Fam Cancer. 2006;5(4):327-35.

21. Laitman $Y$, Vaisman $Y$, Feldman D, Helpman L, Gitly M, Paluch Shimon S, et al. Rates of risk-reducing surgery in Israeli BRCA1 and BRCA2 mutation 
carriers. Clin Genet. 2014;85:68-71 [cited 2019 Sep 2]. Available from: http://www.ncbi.nlm.nih.gov/pubmed/23517234.

22. Heemskerk-Gerritsen BAM, Seynaeve C, Van Asperen CJ, Ausems MGEM Collée JM, Van Doorn HC, et al. Breast cancer risk after salpingo-oophorectomy in healthy BRCA1/2 mutation carriers: revisiting the evidence for risk reduction. J Natl Cancer Inst. 2015;107(5):djv033.

23. Kotsopoulos J, Huzarski T, Gronwald J, Singer CF, Moller P, Lynch HT, et al. Bilateral oophorectomy and breast cancer risk in BRCA1 and BRCA2 mutation carriers. J Natl Cancer Inst. 2017:109.

24. Terry MB, Daly MB, Phillips KA, Ma X, Zeinomar N, Leoce N, et al. Risk-reducing oophorectomy and breast cancer risk across the spectrum of familial risk. J Natl Cancer Inst. 2019;111:331-4 Oxford University Press; [cited 2020 Sep 20]. Available from: /pmc/articles/ PMC6410936/?report=abstract.

\section{Publisher's Note}

Springer Nature remains neutral with regard to jurisdictional claims in published maps and institutional affiliations.
Ready to submit your research? Choose BMC and benefit from:

- fast, convenient online submission

- thorough peer review by experienced researchers in your field

- rapid publication on acceptance

- support for research data, including large and complex data types

- gold Open Access which fosters wider collaboration and increased citations

- maximum visibility for your research: over $100 \mathrm{M}$ website views per year

At BMC, research is always in progress.

Learn more biomedcentral.com/submissions 